\title{
A review of asymptotic procedures in stress analysis: known solutions and their applications
}

\author{
D A Hills*, D Dini, A Magadu and A M Korsunsky \\ Department of Engineering Science, University of Oxford, Oxford, UK
}

\begin{abstract}
A comprehensive review is given of the origins of asymptotic procedures in stress analysis. Specifically, attention is focused on the use of fracture mechanics to characterize the elastic stress state ahead of a crack tip. Analogies are then drawn between this configuration and the stress state adjacent to the apex of a sharp V-notch. Extensions of these asymptotic procedures to bonded and slipping contacts are then considered and it is shown that although power order singularities may be obtained, the solutions are more complicated. Lastly, the use of nested asymptotic procedures are considered in order to account for a small but finite radius at the tip of cracks and notches or at the edge of slipping contacts.
\end{abstract}

Keywords: asymptotic analysis

\section{NOTATION}

$a$

G

$K_{i}$

$(r, \theta)$

$u_{i} \quad$ local displacement field in the $i$ direction $x$ or $y$

$v(x, y)$ local displacement field in Cartesian coordinates

$w$

$(x, y)$

$(\alpha, \beta)$

$\left(\alpha_{1}, \alpha_{2}\right)$

$\kappa$

$\lambda$

$\mu$

$\mu$

$(\rho, \theta)$

$\sigma_{i j}$

$\tau_{i j}$

$\phi(z)$

$\varphi$

$\Psi$

The MS was received on 23 January 2003 and was accepted after revision for publication on 21 July 2004.

* Corresponding author: Department of Engineering Science, University of Oxford, Parks Road, Oxford OX1 3PJ,UK. E-mail: david.hills@, eng.ox.ac.uk

\section{INTRODUCTION}

The most commonly encountered asymptotic procedure in stress analysis is the concept of fracture mechanics and the framework it provides for the prediction of both monotonic fracture loads and the rate of growth of fatigue cracks. As this may be a familiar subject the ideas behind it will be developed in some detail, before going on to more general applications where the same principles apply. In brief, the whole concept of asymptotic analysis relies on being able to focus on some feature, such as the tip of a crack, or the apex of a sharp notch, or the corner of some slipping complete contact, and to recognize that the stress state in the neighbourhood of that feature may be the same (in the sense of having the same spatial distribution of stresses) as all other features having the same local geometry: the influence of remote boundaries forming the shape of the component, may be irrelevant. It follows that a simplified solution, ignoring the presence of all remote boundaries so that the key feature under consideration is present in a semi-infinite domain, may correctly capture that stress state. Thus, as the feature is approached in the finite and semi-infinite bodies the stress states implied by an elastic solution become the same (hence, within certain limits, all crack tips exhibit the same state of stress). This has further implications for the strength of the component if failure originates at the feature; in brief, it shows that a single quantity scaling the state of stress may be used to correlate the failure load in any pair of components having the same local geometry. The most commonly encountered quantity is that employed in 
linear elastic fracture mechanics, viz. the stress intensity factor.

Recent developments in the applications of asymptotic analysis have included the prediction of the fracture strength of notched components and fretting contacts. These will be described, together with the use of nested asymptotic solutions, to permit the effects of various local variations in the geometry to be gauged, and in which the authors have been particularly involved.

\section{FRACTURE MECHANICS}

There are many undergraduate textbooks on fracture mechanics, including those by Broek [1, 2], Ewalds and
Wanhill [3] and Gdoutos [4]. Many elementary approaches to the subject fail to take a strong enough account of why the subject hinges on the stress intensity factor, and recent texts at the first year postgraduate level, taking a more rigorous approach, include another by Gdoutos [5], one by Kanninen and Popelar [6] and another by Broberg [7]. A particularly rigorous and appropriate approach, in the present context, is the one taken by Aliabadi and Rooke [8]. It is not possible to develop the subject ab initio here, but the key elements in the technique will be given.

Consider a crack, of half-length $a$, in an infinite plane, shown in Fig. 1a. Suppose, for the time being, that it is subjected to remote tension, $\sigma_{0}$, in both the $x$ and $y$ directions. A solution is required to the boundary

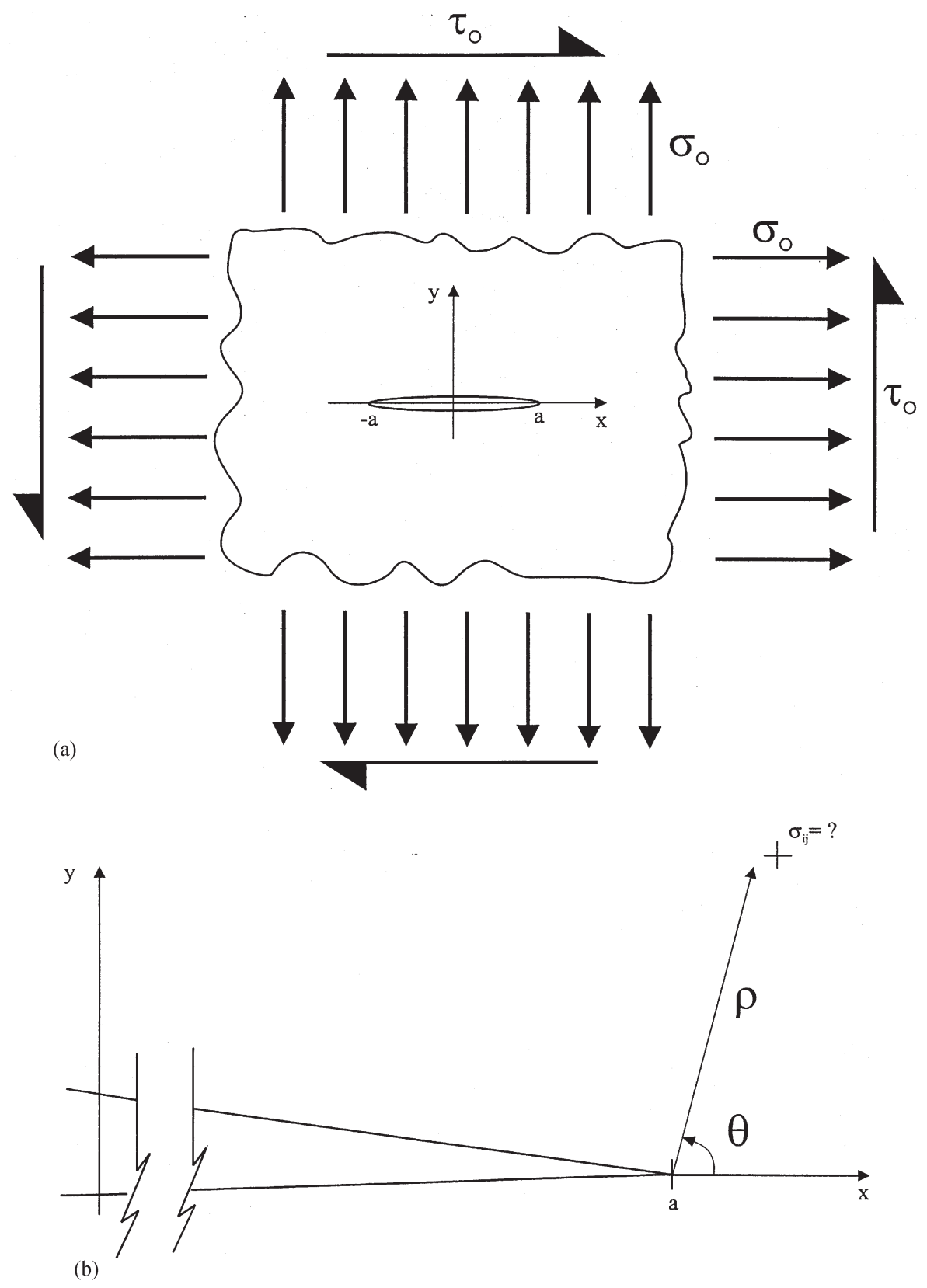

Fig. 1 (a) Crack in an infinite plane. (b) Local coordinates at the crack tip 
value problem

$$
\begin{aligned}
& \left\{\begin{array}{l}
\sigma_{x x} \\
\sigma_{y y} \\
\tau_{x y}
\end{array}\right\} \rightarrow\left\{\begin{array}{c}
\sigma_{0} \\
\sigma_{0} \\
0
\end{array}\right\} \quad \text { Limit }|x, y| \rightarrow \infty \\
& \left\{\begin{array}{l}
\sigma_{y y} \\
\tau_{x y}
\end{array}\right\} \rightarrow\left\{\begin{array}{l}
0 \\
0
\end{array}\right\} \quad \text { Limit }|y| \rightarrow 0,|x|<a
\end{aligned}
$$

This problem was first solved by Westergaard [9], who showed that the solution was given by

$$
\phi(z)=\frac{\sigma_{0} z}{\sqrt{z^{2}-a^{2}}}
$$

where $\phi(z)$ is a complex stress function and

$$
z=x+\mathrm{i} y
$$

The corresponding stresses are then defined as

$$
\begin{aligned}
\sigma_{x x} & =\operatorname{Re}[\phi(z)]-y \operatorname{Im}\left[\phi^{\prime}(z)\right] \\
\sigma_{y y} & =\operatorname{Re}[\phi(z)]+y \operatorname{Im}\left[\phi^{\prime}(z)\right] \\
\tau_{x y} & =-y \operatorname{Re}\left[\phi^{\prime}(z)\right]
\end{aligned}
$$

where $\phi^{\prime}(z)$ is the first-order derivative of $\phi(z)$. The local displacement field is given by

$$
\begin{aligned}
& 2 \mu u(x, y)=\frac{\kappa-1}{2} \operatorname{Re}[\bar{\phi}(z)]-y \operatorname{Im}[\phi(z)] \\
& 2 \mu v(x, y)=\frac{\kappa-1}{2} \operatorname{Im}[\bar{\phi}(z)]-y \operatorname{Re}[\phi(z)]
\end{aligned}
$$

where $\bar{\phi}(z)$ is the complex conjugate of $\phi(z), \mu$ is the modulus of rigidity, $\kappa$ is Kolosov's constant $[=3-4 \nu$ in plane strain and $(3-\nu) /(1+\nu)$ in plane stress] and $\nu$ is Poisson's ratio.

Now a new local polar coordinate set, $\zeta=z-a$, is set up where $\zeta(\rho, \theta)$ is positioned at the crack tip (see Fig. 1b). The stress function may be written in the form $\phi(\zeta)=\sigma_{0}(\zeta+a) /[\zeta(\zeta+2 a)]^{1 / 2}$. By expanding this expression using the binomial theorem, and taking the lead term, it can be seen that the state of stress for small $\rho / a$ is given approximately by

$$
\left\{\begin{array}{c}
\sigma_{x x} \\
\sigma_{y y} \\
\tau_{x y}
\end{array}\right\}=\frac{K_{\mathrm{I}}}{\sqrt{2 \pi \rho}}\left\{\begin{array}{c}
\cos (\theta / 2)[1-\sin (\theta / 2) \sin (3 \theta / 2)] \\
\cos (\theta / 2)[1+\sin (\theta / 2) \sin (3 \theta / 2)] \\
\sin (\theta / 2) \cos (\theta / 2) \cos (3 \theta / 2)
\end{array}\right\},
$$

$$
\rho \ll a
$$

where

$$
K_{\mathrm{I}}=\sigma_{0} \sqrt{\pi a}
$$

For completeness the local displacement field is also recorded, which is given by

$$
\left\{\begin{array}{l}
u_{x} \\
u_{y}
\end{array}\right\}=\left(\frac{K_{\mathrm{I}}}{2 \mu}\right) \sqrt{\frac{\rho}{2 \pi}}\left\{\begin{array}{c}
\cos (\theta / 2)(\kappa-\cos \theta) \\
\sin (\theta / 2)(\kappa-\cos \theta)
\end{array}\right\}
$$

This solution is far more than the asymptotic stress state relevant to the case of uniform remote loading. Provided that the value of $K_{\mathrm{I}}$ is chosen correctly, equation (7) gives the stress at the apex of any crack, subject to remote loading, which simply tends to open the crack $\left(\sigma_{y y}>0\right.$, $\tau_{x y}=0$ on $\theta=0, \rho>0$ ), and is known in the jargon as mode I loading. As part of the analysis for a wedge or notch (section 3), this solution, i.e. equation (7), will be shown to correspond to a special case [equation (33)].

A second, independent, solution may be found by an analogous method (see Gdoutos [5] for details) for the case when the crack in an infinite plane is subject to remote shear, $\tau_{x y}=\tau_{0}$, i.e.

$$
\left\{\begin{array}{c}
\sigma_{x x} \\
\sigma_{y y} \\
\tau_{x y}
\end{array}\right\} \rightarrow\left\{\begin{array}{c}
0 \\
0 \\
\tau_{0}
\end{array}\right\} \quad \text { Limit }|x, y| \rightarrow \infty
$$

The corresponding potential is given by

$$
\phi(z)=-\frac{i \tau_{0} z}{\sqrt{z^{2}-a^{2}}}
$$

This complex stress function is connected to the stress field by the equations

$$
\begin{aligned}
\sigma_{x x} & =2 \operatorname{Re}[\phi(z)]-y \operatorname{Im}\left[\phi^{\prime}(z)\right] \\
\sigma_{y y} & =y \operatorname{Im}\left[\phi^{\prime}(z)\right] \\
\tau_{x y} & =-\operatorname{Im}[\phi(z)]-y \operatorname{Re}\left[\phi^{\prime}(z)\right]
\end{aligned}
$$

while the local displacement field is given by

$$
\begin{aligned}
& 2 \mu u(x, y)=\frac{\kappa+1}{2} \operatorname{Re}[\bar{\phi}(z)]-y \operatorname{Im}[\phi(z)] \\
& 2 \mu v(x, y)=\frac{\kappa-1}{2} \operatorname{Im}[\bar{\phi}(z)]-y \operatorname{Re}[\phi(z)]
\end{aligned}
$$

Here the asymptotic solution is given by

$$
\left\{\begin{array}{c}
\sigma_{x x} \\
\sigma_{y y} \\
\tau_{x y}
\end{array}\right\}=\frac{K_{\mathrm{II}}}{\sqrt{2 \pi \rho}}\left\{\begin{array}{c}
-\sin (\theta / 2)[2+\cos (\theta / 2) \cos (3 \theta / 2)] \\
\sin (\theta / 2) \cos (\theta / 2) \cos (3 \theta / 2) \\
\cos (\theta / 2)[1-\sin (\theta / 2) \sin (3 \theta / 2)]
\end{array}\right\},
$$

where

$$
K_{\mathrm{II}}=\tau_{0} \sqrt{\pi a}
$$

Again, for completeness the local displacement field is also recorded as

$$
\left\{\begin{array}{l}
u_{x} \\
u_{y}
\end{array}\right\}=\left(\frac{K_{\mathrm{II}}}{2 \mu}\right) \sqrt{\frac{\rho}{2 \pi}}\left\{\begin{array}{l}
2+\kappa+\cos \theta \\
2-\kappa-\cos \theta
\end{array}\right\}
$$


As before, this solution is of more general relevance, as it applies whenever the remote loading tends simply to shear the crack $\left(\sigma_{y y}=0, \tau_{x y}>0\right.$ on $\left.\theta=0, \rho>0\right)$, and is known in the jargon as mode II loading. Again, the analysis for a wedge or notch will show this as a special case.

There is a third form of loading that may arise, known as 'anti-plane' deformation. In the notation of Fig. 1 this occurs when the remote loading is in the form of a shear into or out of the plane, rather like tearing a book, and the remote boundary condition is now $\tau_{y z}=\tau_{z 0}$. The corresponding solution is given by

$$
\phi(z)=\frac{\tau_{z 0} z}{\sqrt{z^{2}-a^{2}}}
$$

where, in this kind of loading, the non-zero stresses are given by

$$
\begin{aligned}
\tau_{z x} & =\operatorname{Im}\left[\phi^{\prime}(z)\right] \\
\tau_{y z} & =\operatorname{Re}\left[\phi^{\prime}(z)\right]
\end{aligned}
$$

while the transverse direction (normal to the $x y$ plane) displacement, $w$, is given by

$$
w=\frac{1}{\mu} \operatorname{Im}[\phi(z)]
$$

The asymptotic solution then takes the form

$$
\left\{\begin{array}{c}
\tau_{x z} \\
\tau_{y z}
\end{array}\right\}=\frac{K_{\text {III }}}{\sqrt{2 \pi \rho}}\left\{\begin{array}{c}
-\sin (\theta / 2) \\
\cos (\theta / 2)
\end{array}\right\}
$$

where

$$
K_{\mathrm{III}}=\tau_{z 0} \sqrt{\pi a}
$$

and the displacement is given by

$$
w=\left(\frac{2 K_{\mathrm{III}}}{\mu}\right) \sqrt{\frac{\rho}{2 \pi}} \sin (\theta / 2)
$$

\subsection{Physical relevance of the solution}

The solutions obtained above have been discovered by several analysts and by different routes. Each gives an independent contribution to the singular elastic stress field adjacent to a crack tip. As the solutions are elastic they may be applied together, from the principle of superposition, to give a 'mixed mode' crack tip field. Note that, in terms of a series expansion of the state of stress, the next term in the series is one where the stress state varies as $\sqrt{\rho}$ for all three components of loading, i.e. next in sequence to equations (7), (14) and (20). The contributions from the second terms are therefore bounded, but the application of a remote load parallel with the crack faces, $\sigma_{\mathrm{T}}$, is, to a first-order approximation, unaffected by the presence of the crack. It therefore gives rise to a contribution to the local stress state that is less significant than the first-order singular terms derived, but may be more important than the secondorder bounded terms, at small but finite distances from the crack tip. It is known, in fracture mechanics jargon, as the 'T-stress'.

Monotonic fracture of a highly brittle material subject to remote tension applied perpendicular to the faces of the crack is governed by the celebrated Griffith criterion $[4, \mathbf{1 0}]$. This simply states that a crack cannot extend until the strain energy which is released, $\mathrm{d} U$, under remote controlled-displacement conditions, when the crack extends by an amount $\mathrm{d} a$, is at least as great as that needed to form the new crack surfaces, $\gamma$ per unit area, assumed to be a material property. A quantity $G$ is introduced, the strain energy release rate or generalized crack extension force, defined by $G=\mathrm{d} U / \mathrm{d} a$, and crack extension occurs when

$$
G=2 \gamma
$$

It may be shown that $G$ is related to the mode I stress intensity factor by evaluating the energy released when the crack extends by a small amount. This is found by evaluating the work done in closing the crack, over an infinitesimal distance, $\delta$, giving

$$
G=2 \operatorname{Limit}\left[\frac{1}{2 \delta} \int_{0}^{\delta} \sigma_{y y}(\delta-\rho) v(\rho) \mathrm{d} r\right]
$$

where, because the distance $\delta$ is small compared with the crack length so that the integral is evaluated within the region dominated by the singular region, the traction is given by $\sigma_{y y}=K_{\mathrm{I}} / \sqrt{2 \pi(\delta-\rho)}$ and $v(r)=$ $K_{\mathrm{I}}[(\kappa+1) / 2 \mu] \sqrt{\rho / 2 \pi}$. This is one part of the so-called Irwin $K-G$ relation, and is

$$
G=\frac{(\kappa+1) K_{\mathrm{I}}^{2}}{8 \mu}
$$

It demonstrates that the scaling factor for the asymptotic crack tip stress field, the stress intensity factor, controls fracture for a brittle material.

The asymptotic field has greater relevance than this, however. Firstly, in the presence of a very modest amount of plasticity, the assumption that the stress intensity factor controls fracture is adopted as a hypothesis, simply because the state of stress in the fracture zone is itself proportional to $K_{\mathrm{I}}$. Thus, it is argued that there is a material property that represents the critical value of the stress intensity factor at which fracture occurs. This 'material property' is dependent on the degree of transverse constraint but is substantially constant under plane strain conditions, when it is known as the fracture toughness and given the symbol $K_{\mathrm{IC}}$. This hypothesis has 
been experimentally tested and been shown to be true, provided that the plasticity which is present at the crack tip is modest.

The relationship between the strain energy released when the crack extends and the stress intensity factors present may be extended to modes II and III loading, using, again, an infinitesimal increase in crack length as the vehicle. The following complete form for the relationship between $G$ and $K_{\mathrm{I}}, K_{\mathrm{II}}, K_{\mathrm{III}}$ emerges

$$
G=\frac{(\kappa+1)\left(K_{\mathrm{I}}^{2}+K_{\mathrm{II}}^{2}\right)}{8 \mu}+\frac{K_{\mathrm{III}}^{2}}{2 \mu}
$$

It is postulated that the crack will extend when the value of $G$ reaches a critical value, with contributions from all three forms of remote load, although this extension to the original concept is not universally accepted (see Sih $[11,12])$. The point is made, however, that the elastic singular solution contributions must again control the complete fracture process.

A consideration of the relevance of the crack tip stress intensity factors to the problem of crack propagation under cyclic loading will now be considered. Crack propagation occurs by the local exhaustion of plasticity in a small region (the 'process zone') at the tip of the crack. It is not known what attributes of the local stress or strain field actually control the exhaustion process, but it is clear that they must be associated with the local plastic stress and strain. These quantities are themselves difficult to quantify, because they require the solution of a fullyfledged elastic-plastic problem: indeed, even if it were possible to find the true local values of stress and strain, that would still not represent a complete solution to the problem, because there is no universal criterion for the exhaustion of ductility. However, the argument is made that, if the process zone is small compared with an elastic hinterland in which the singular solution itself dominates, all the relevant quantities controlling the inner process zone are themselves determined by a single scaling variable, viz. the stress intensity factor. The concept of 'small-scale yielding', which must be satisfied for crack growth to be controlled by the stress intensity factor, is precisely this. In fact, there are stronger requirements on the nature of the stress state that the crack tip experiences: (a) if the T-stress is significant in proportion to the singular field at the position of the process zone front, that may have an influence on the fatigue performance; (b) if the crack tip is experiencing combined modes loading, each non-zero mode contributes to the local stress field and, if the ratio between the stress intensity factors varies during the loading cycle (the so-called 'non-proportional loading' regime), the crack may propagate in a way that is difficult to predict; the latter is the subject of current research [13]. Lastly, it is known that it is the range of plastic strain (rather than its maximum value) which is principally responsible for the rate of crack extension. Thus, the key parameters at the crack tip may be thought of as functions of both $K_{\max }$ and $K_{\min }$. These are not the two best choices of characteristic parameter, however: it is better to use the range of stress intensity experienced ( $\left.\Delta K=K_{\max }-K_{\min }\right)$ and something quantifying the average value of the local loads. The $R$ ratio is normally chosen for this, which may be defined either classically using the nominal stress present in the crack's absence or, for consistency, in terms of the stress intensity factors as $R=K_{\min } / K_{\max }$. Note that this definition requires that $K_{\min } \geqslant 0$, and hence $R \geqslant 0$. The crack growth rate is a strong function of $\Delta K$ and a weak function of $R$. It is emphasized again here that the actual size of the plastic zone is necessarily very small for the principles behind elastic fracture mechanics to hold. The plastic zone must be much smaller than the region over which the singular term solutions dominate the state of stress.

Note that the fracture of heterogeneous materials may not introduce any fundamentally new principles, providing that the size of each characteristic phase or component is large compared with the process zone size, and the fracture principles cited may be applied on a pointwise basis. The presence of interfaces between those phases may, however, introduce further problems, because the singularities induced there may be different. This issue will be addressed in section 5 .

\section{NOTCH PROBLEM}

The results reviewed above, abstracted from standard fracture mechanics theory, may be arrived at in another way, involving not the asymptotic expansion of the local stress field around the tip of a crack in a particular geometry but from a solution constructed in an infinite domain. The best way to do this is to consider a semiinfinite notch or wedge (Fig. 2) of internal angle $2 \varphi$ and to deduce the stress field in the neighbourhood of the notch tip. This is a well-posed problem, first solved by Williams [14], and the general results obtained are of practical relevance, as they may be used to define characteristic singular local fields. As a special case, by making the internal notch half-angle equal to $\pi$, the characteristic stress fields derived above may be deduced directly. The details of the algebra needed may be found in the books by Barber [15] and by Aliabadi and Rooke $[\mathbf{8}]$, and here only an outline of the method will be reproduced. The nomenclature used in fracture mechanics relating to the three independent modes of crack tip loading is adopted for notches. In-plane loading will therefore be considered first. Polar coordinates $(r, \theta)$ centred on the notch apex (Fig. 2) are appropriate and the biharmonic equation is then given by

$$
\left[\frac{\partial^{2}}{\partial r^{2}}+\frac{1}{r} \frac{\partial}{\partial r}+\frac{1}{r^{2}} \frac{\partial^{2}}{\partial \theta^{2}}\right]^{2} \Psi=0
$$




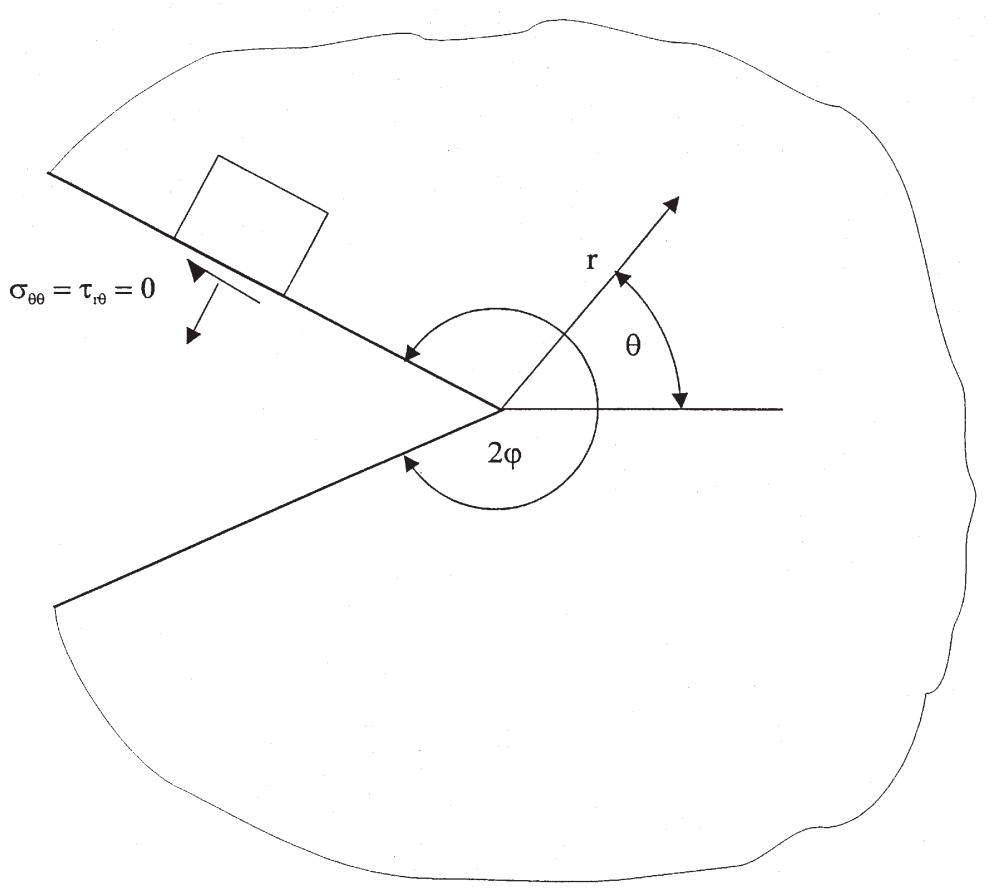

Fig. 2 Semi-infinite wedge of notch

where the stress components are related to the stress function, $\Psi$, by

$$
\begin{aligned}
\sigma_{r r} & =\frac{1}{r} \frac{\partial \Psi}{\partial r}+\frac{1}{r^{2}} \frac{\partial^{2} \Psi}{\partial \theta^{2}} \\
\sigma_{\theta \theta} & =\frac{\partial^{2} \Psi}{\partial r^{2}} \\
\tau_{r \theta} & =\frac{1}{r^{2}} \frac{\partial \Psi}{\partial \theta}-\frac{1}{r} \frac{\partial^{2} \Psi}{\partial r \partial \theta}
\end{aligned}
$$

If it is assumed, pro tem, that there are tractions varying in a power series along the faces of the notch, the form of the above equations would suggest an investigation of a variables-separable solution of the form

$$
\Psi=r^{\lambda+1} F(\theta)
$$

If this is substituted into the biharmonic equation the $r$ variation is seen to be satisfied, and $F(\theta)$ emerges as

$$
\begin{aligned}
F(\theta)= & A_{1} \cos (\lambda-1) \theta+A_{2} \cos (\lambda+1) \theta \\
& +A_{3} \sin (\lambda-1) \theta+A_{4} \sin (\lambda+1) \theta
\end{aligned}
$$

This solution is now substituted into equation (28) to obtain the stresses and the traction components are set on the faces $\theta= \pm \varphi$ to 0 . This then yields the following set of two pairs of uncoupled simultaneous homogeneous equations:

$$
\begin{gathered}
{\left[\begin{array}{ccc}
\cos (\lambda-1) \varphi & \cos (\lambda+1) \varphi \\
\Xi \sin (\lambda-1) \varphi & \sin (\lambda+1) \varphi \\
0 & 0 & 0 \\
0 & 0 & 0 \\
0 & \sin (\lambda+1) \varphi \\
\sin (\lambda-1) \varphi & \cos (\lambda+1) \varphi
\end{array}\right]\left\{\begin{array}{l}
A_{1} \\
A_{2} \\
A_{3} \\
A_{4}
\end{array}\right\}} \\
\quad \Xi \cos (\lambda-1) \varphi \\
=\left\{\begin{array}{l}
0 \\
0 \\
0 \\
0
\end{array}\right\}
\end{gathered}
$$

where $\Xi=(\lambda-1) /(\lambda+1)$. The first two equations correspond to a symmetric solution while the second two are antisymmetric. The solution of these equations requires the determinants of the separate pairs of equations to vanish, leading to the following eigenequations:

$$
\sin \lambda \varphi \pm \lambda \sin 2 \varphi=0
$$

where the symmetric solution is associated with the + sign and the antisymmetric solution with the - sign. The corresponding eigenvalues are plotted in Fig. 3. It should be recalled that the stress state varies as $r^{\lambda-1}$, so the stress state is bounded when $\lambda-1>0$ and is 


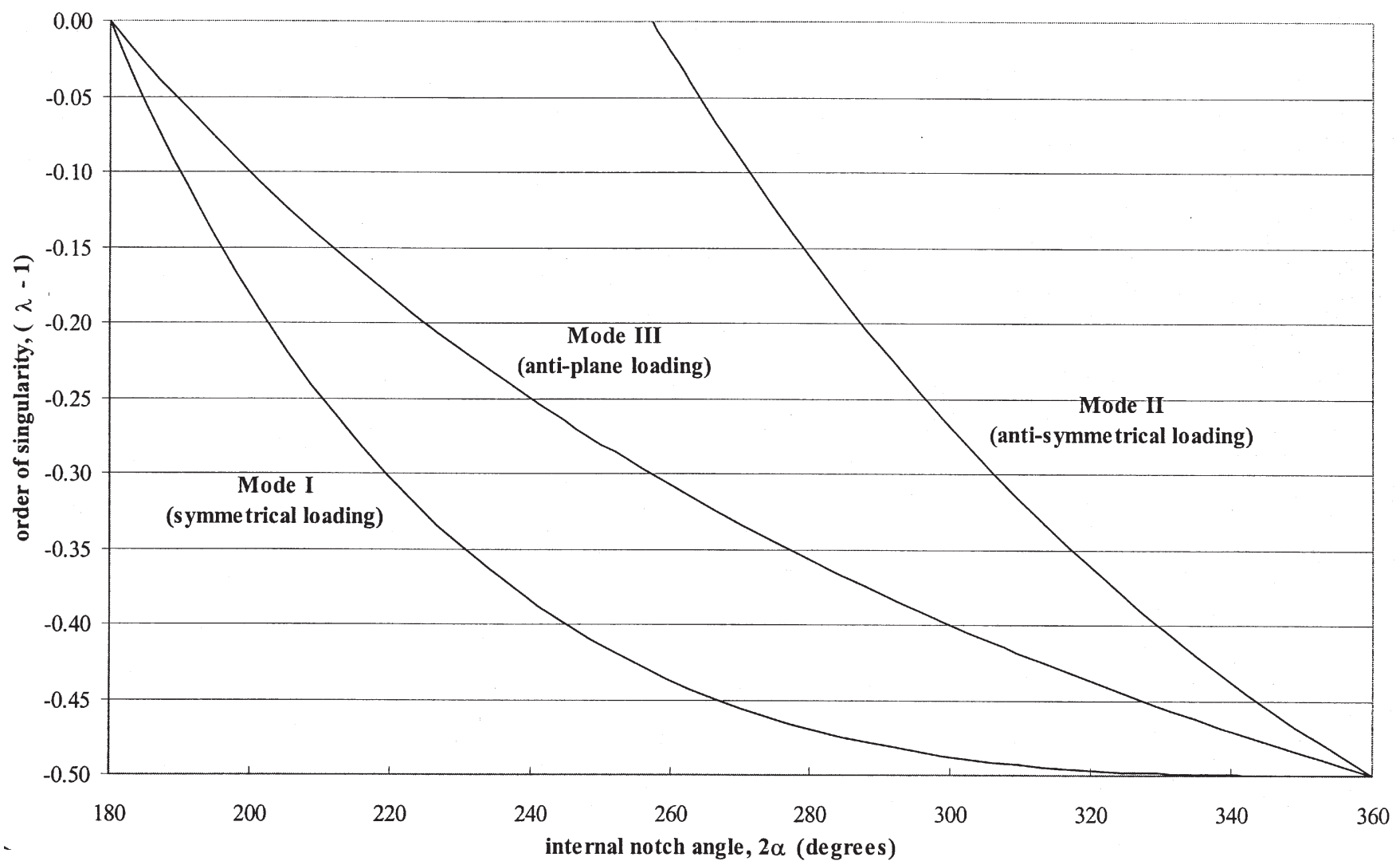

Fig. 3 Order of singularity, $\lambda-1$, at a notch apex

singular when $\lambda-1<0$. The figure displays the results in the practically important singular region and indicates that the symmetric solution is singular if $\varphi>\pi / 2$ radians, while the antisymmetric solution is singular only if $\varphi>128.7^{\circ}$. Further, the symmetric solution is always more strongly singular than the antisymmetric one, for any given notch, so that, unless special precautions are taken to suppress the symmetric mode (in practice this rarely happens; indeed, it is very hard to contrive), the symmetric solution will dominate the solution for sufficiently small $r .^{\dagger}$ If the eigenvalue is back-substituted into the relevant pair of equations in equation (31) the ratio $A_{1} / A_{2}$ (symmetric solution) or $A_{3} / A_{4}$ (antisymmetric solution) may be found. These then serve to fix the spatial distribution of stress with the polar angle $\theta$, allowing the overall solutions to be written in the form

$$
\sigma_{i j}(r, \theta)=K_{\mathrm{I}}^{*} r^{\lambda_{\mathrm{I}}-1} F_{\mathrm{I}}(\theta)+K_{\mathrm{II}}^{*} r^{\lambda_{\mathrm{II}}-1} F_{\mathrm{II}}(\theta)
$$

where the multiplicative factors $K_{n}^{*}$ take on the rôle of generalized stress intensity factors. Their value is, in practice, found by collocating the asymptotic solution with the local stress state given by the full solution, normally found by the finite element method of Tur et al.

\footnotetext{
${ }^{\dagger}$ The asymptotic solution is usually used to infer properties of the process zone. As this is of finite extent the contribution from the antisymmetric term may be significant, especially if the multiplicative factors in the solution are comparable.
}

[16]. For example, under pure mode I loading a plot is made of $\sigma_{\theta \theta}(r, 0) r^{1-\lambda_{\mathrm{I}}}$ against $r$. As $r \rightarrow 0, \sigma_{\theta \theta} \rightarrow \infty$ but their product is finite and equal to $K_{\mathrm{I}}^{*}$.

Note that if $\varphi$ is set to $\pi$ in equation (32) above and the resulting eigenvalue $(\lambda=0.5)$ is back-substituted into equation (31) for the case of symmetric loading, equation (33) becomes identical to equation (14).

The singular solutions for notches are less well known than their specialized counterparts, the singular crack-tip solutions, described in section 2. It must be emphasized that the mode I and II terms do not necessarily contribute equally to the magnitude of the local stress and the former dominates. Potentially, these solutions take on a function analogous to the crack tip stress fields, but they have found rather less practical application so far. This is partly because sharp notches are always avoided, if at all possible, and partly because, even when the geometry does arise, there is very often at least some notional radius present at the notch root that may invalidate the solution. This point will be addressed in a later section. There remains significant practical objections to the use of the generalized stress intensity factors as controllers of the notch monotonic fracture strength, not least because there is no release of energy to propel the subsequent crack when the notch forms a crack [17]. However, there is less objection to their use as controllers of the size of the local process zone and hence as the basis of a crack initiation criterion. 
A third asymptotic solution applies when the notch root is loaded in anti-plane shear (the mode III solution in fracture mechanics nomenclature), although the authors have not been able to find a reference to it in the literature. Its derivation is straightforward and a beginning is made by writing the governing differential equation for this class of problem, viz.

$$
\Delta F(r, \theta)=0
$$

where

$$
\begin{aligned}
\tau_{r z} & =-\frac{\partial F}{\partial r} \\
\tau_{z \theta} & =\frac{1}{r} \frac{\partial F}{\partial \theta}
\end{aligned}
$$

It is assumed that $F(r, \theta)$ may be written in the variablesseparable form $F(r, \theta)=r^{\lambda} T(\theta)$, so that

$$
\Delta F(r, \theta)=r^{\lambda-2}\left(\lambda^{2} T+\frac{\partial^{2} T}{\partial \theta^{2}}\right)
$$

from which

$$
F(r, \theta)=r^{\lambda}\left(c_{1} \cos \lambda \theta+c_{2} \sin \lambda \theta\right)
$$

and the only non-zero stresses are given by

$$
\begin{gathered}
\tau_{r z}=-\frac{\partial F}{\partial r}=\lambda r^{\lambda-1}\left(c_{1} \cos \lambda \theta+c_{2} \sin \lambda \theta\right) \\
\tau_{z \theta}=\frac{1}{r} \frac{\partial F}{\partial \theta}=\lambda r^{\lambda-1}\left(-c_{1} \sin \lambda \theta+c_{2} \cos \lambda \theta\right)
\end{gathered}
$$

(a)

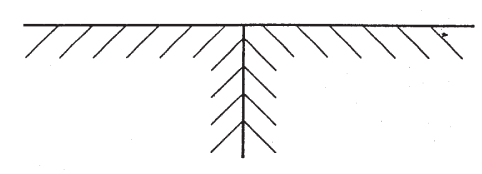

(c)

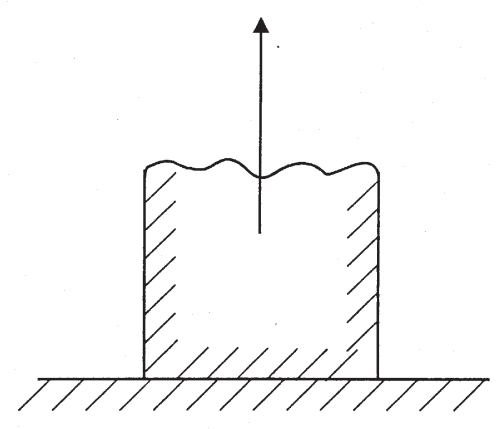

(e)

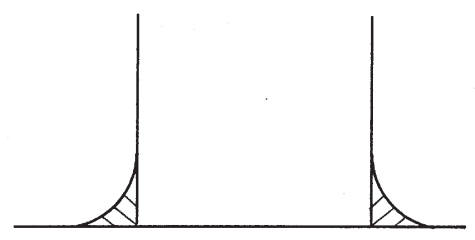

Now set the traction component of stress $\left(\tau_{z \theta}\right)$ to zero on the faces $\theta= \pm \varphi$, to give the following characteristic equation:

$$
\sin 2 \lambda \varphi=0
$$

from which the relevant eigenvalue is given by

$$
\lambda=\frac{\pi}{2 \varphi}
$$

Back-substituting, as before, yields the eigenfunction and hence the spatial distribution of the non-zero shear stresses, which take on a particularly simple form. The solution (see Fig. 3) is very similar in behaviour to the symmetrical solution for the in-plane problem. It shows that all re-entrant wedges are singular, with, as expected, the strongest singularity (square root) when the wedge is folded to form a crack, i.e. $2 \varphi=2 \pi$ radians. For any given wedge angle the mode III singularity is weaker than the mode I solution, but stronger than the mode II solution.

\section{BONDED COMPONENTS}

When two elastically similar bodies are bonded together, there may be an abrupt change in stiffness across the bond line. Where the bond line meets the free surface a singular state of stress may arise, depending on the local contact geometry. Some simple cases are shown in Fig. 4. They include the butt joint, where the interface line meets the free surface normally (Fig. 4a), and this is seen to be a special case of the scarf joint (Fig. 4b). In these cases

(b)
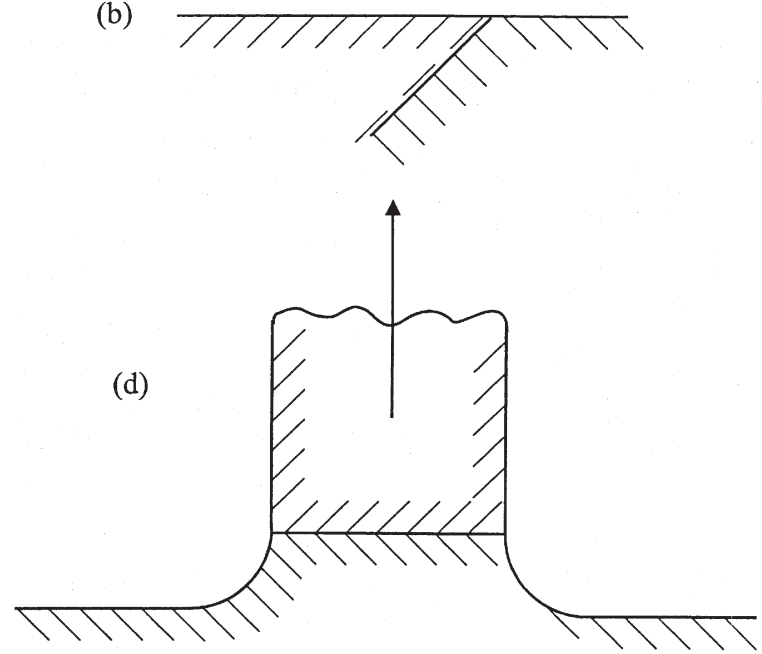

(f)

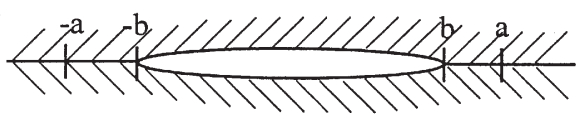

Fig. 4 Examples of bonded components for idealized geometries 

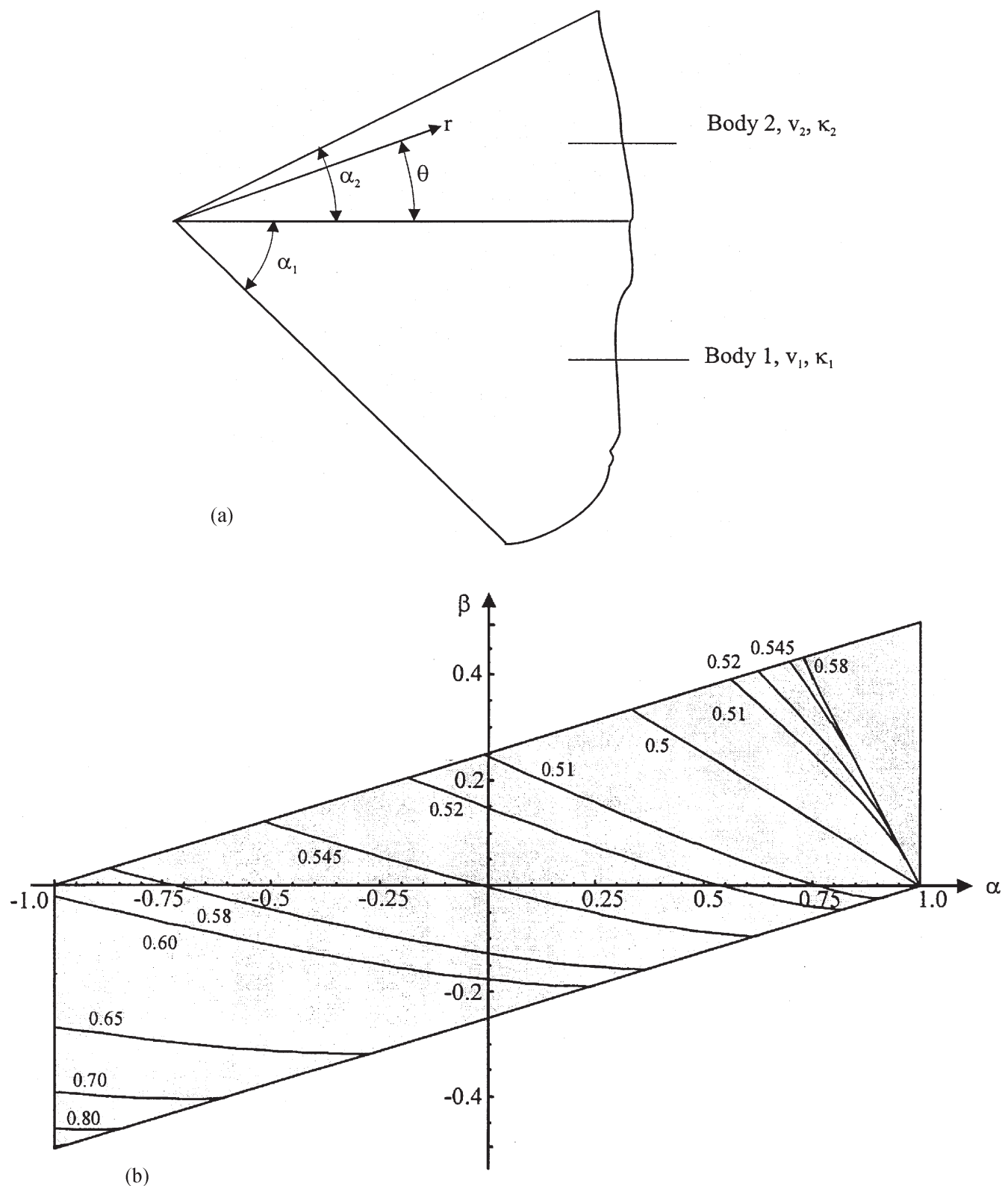

Fig. 5 (a) Bonded elastically dissimilar wedges. (b) Dundurs' parallelogram for $\alpha_{1}=\pi$ and $\alpha_{2}=\pi / 2$

the free surface is locally straight, whereas locally attaching a block also produces a geometric discontinuity (Fig. $4 c)$. If it is found that this gives rise to a very severe stress intensity it is possible to modify the local geometry to produce a pedestal mounting (Fig. 4d), and in the case of a soldered or brazed joint the weld/braze material may produce a local feature such as that shown in Fig. 4(e). Problems of this kind were studied by Bogy [18], by representing the feature as two bonded elastic semiinfinite wedges (Fig. 5a), so that the salient features of the geometry and interface may be included. The underlying problem that has to be studied is therefore that of two elastically dissimilar bonded wedges, as shown in Fig. 5a. The solutions to such problems clearly depend on a total of four elastic constants (two for each material) and, indeed, in the original paper all four constants appeared in the solution. Subsequently, Dundurs [19] showed that the key features of the solution depended on only two quantities, which have now entered the literature as the 'Dundurs' constants'. These are

$$
\begin{aligned}
& \alpha=\frac{\left(\mu_{2} / \mu_{1}\right)\left(\kappa_{1}+1\right)-\left(\kappa_{2}+1\right)}{\left(\mu_{2} / \mu_{1}\right)\left(\kappa_{1}+1\right)+\left(\kappa_{2}+1\right)} \\
& \beta=\frac{\left(\mu_{2} / \mu_{1}\right)\left(\kappa_{1}-1\right)-\left(\kappa_{2}-1\right)}{\left(\mu_{2} / \mu_{1}\right)\left(\kappa_{1}+1\right)+\left(\kappa_{2}+1\right)}
\end{aligned}
$$

where the subscripts 1 and 2 refer to the bodies shown in Fig. 5a. Bogy subsequently re-examined his results [20] and published them in a more comprehensive form, making use of the 'Dundurs' parallelogram diagram' (Fig. 5b). If Poisson's ratio for both materials lies in the 
range $0 \leqslant \nu_{i} \leqslant \frac{1}{2}$, all physically acceptable combinations of materials must lie within this parallelogram. In particular, Fig. $5 \mathrm{~b}$ shows the values of $\lambda$ to be found below, relating to Fig. 5a, for the special case $\alpha_{1}=\pi$ and $\alpha_{2}=\pi / 2$. Although the solution method used by Bogy was slightly different, a direct method of attack, based on a variation of the technique employed by Williams, would seem to be the most efficient way to derive the results. For each wedge, a variables-separable solution of the kind employed in section 3 is written down. Subscripts 1 and 2 are added for the two bodies and it is supposed that the included angles of each are $\alpha_{1}(<0)$ and $\alpha_{2}(>0)$, with the line $\theta=0$ denoting the interface (Fig. 5). In equation (28) a start was made by displaying the stress-stress function relationships, in order to be able to write down the traction components. The displacement to stress function relationships are now additionally required, in order to be able to develop the continuity conditions across the interface. These are

$$
\begin{gathered}
\frac{\partial u_{r}}{\partial r}=\frac{1}{2 \mu}\left[\frac{1}{r} \frac{\partial \Psi}{\partial r}+\frac{1}{r^{2}} \frac{\partial^{2} \Psi}{\partial r^{2}}-\left(\frac{3-\kappa}{4}\right) \nabla^{2} \Psi\right] \\
\frac{\partial u_{\theta}}{\partial r}-\frac{u_{\theta}}{r}+\frac{1}{r} \frac{\partial u_{r}}{\partial r}=\frac{1}{\mu}\left[-\frac{1}{r} \frac{\partial^{2} \Psi}{\partial r \partial \theta}+\frac{1}{r^{2}} \frac{\partial \Psi}{\partial \theta}\right]
\end{gathered}
$$

As before, the free outer faces are traction free, so that, on $\theta=\alpha_{1}, \alpha_{2}$, the condition $\sigma_{\theta \theta}=\tau_{r \theta}=0$ is required, On the interface $\theta=0$ the following continuity conditions apply:

$$
\begin{aligned}
& \sigma_{\theta \theta 1}=\sigma_{\theta \theta 2} \\
& \tau_{r \theta 1}=\tau_{r \theta 2} \\
& u_{\theta 1}=u_{\theta 2}
\end{aligned}
$$

and, in addition, there is no relative radial displacement, so that

$$
u_{r 1}-u_{r 2}=0
$$

These eight conditions give rise to a homogeneous set of equations for the pair of wedges and the corresponding eigenvalue problem permits the nature of the exponent in the expressions for the stresses to be determined. This is very much more complicated than the monolithic case and the eigenequation will not be derived here. Instead, it will merely be quoted; the determinant that is required to vanish is

$$
\begin{aligned}
& \Delta\left(\alpha_{1}, \alpha_{2}, \alpha, \beta ; \lambda\right) \\
&= A\left(\alpha_{1}, \alpha_{2} ; \lambda\right) \beta^{2} \\
&+2 B\left(\alpha_{1}, \alpha_{2} ; \lambda\right) \alpha \beta+C\left(\alpha_{1}, \alpha_{2} ; \lambda\right) \alpha^{2} \\
&+2 D\left(\alpha_{1}, \alpha_{2} ; \lambda\right) \beta+2 E\left(\alpha_{1}, \alpha_{2} ; \lambda\right) \alpha \\
&+F\left(\alpha_{1}, \alpha_{2} ; \lambda\right)
\end{aligned}
$$

where

$$
\begin{aligned}
A\left(\alpha_{1}, \alpha_{2} ; \lambda\right)= & 4 K\left(\lambda, \alpha_{1}\right) K\left(\lambda, \alpha_{2}\right) \\
B\left(\alpha_{1}, \alpha_{2} ; \lambda\right)= & 2 \lambda^{2} \sin ^{2}\left(\alpha_{1}\right) K\left(\lambda, \alpha_{2}\right) \\
& +2 \lambda^{2} \sin ^{2}\left(\alpha_{2}\right) K\left(\lambda, \alpha_{1}\right) \\
C\left(\alpha_{1}, \alpha_{2} ; \lambda\right)= & 4 \lambda^{2}\left(\lambda^{2}-1\right) \sin ^{2}\left(\alpha_{1}\right) \sin ^{2}\left(\alpha_{2}\right) \\
& +K\left[\lambda,\left(\alpha_{1}-\alpha_{2}\right)\right] \\
D\left(\alpha_{1}, \alpha_{2} ; \lambda\right)= & 2 \lambda^{2}\left[\sin ^{2}\left(\alpha_{1}\right) \sin ^{2}\left(\lambda \alpha_{2}\right)\right. \\
& \left.-\sin ^{2}\left(\alpha_{2}\right) \sin ^{2}\left(\lambda \alpha_{1}\right)\right] \\
E\left(\alpha_{1}, \alpha_{2} ; \lambda\right)= & -D\left(\alpha_{1}, \alpha_{2} ; \lambda\right)+K\left(\lambda, \alpha_{2}\right)-K\left(\lambda, \alpha_{1}\right) \\
F\left(\alpha_{1}, \alpha_{2} ; \lambda\right)= & K\left[\lambda,\left(\alpha_{1}+\alpha_{2}\right)\right]
\end{aligned}
$$

and

$$
K(\lambda, s)=\sin ^{2}(\lambda s)-\lambda^{2} \sin ^{2}(s)
$$

The roots of this determinant govern the behaviour of the state of stress in the following way. If $\lambda_{1}$ is the root of $\Delta$ having the smallest real part in the strip $0<\operatorname{Re}(\lambda) \leqslant 1$, then

$$
\begin{aligned}
& \sigma_{i j}= \\
& \begin{cases}O\left(r^{\lambda_{1}-1}\right) & \text { if } \lambda_{1} \text { real } \\
O\left[r^{\lambda_{1}-1} \cos \left(\lambda_{1} \log r\right)\right] \quad \text { or } \quad O\left[r^{\lambda_{1}-1} \sin \left(\lambda_{1} \log r\right)\right] \\
& \text { if } \lambda_{1}=\xi_{1}+\mathrm{i} \eta_{1} \text { is complex } \\
O(\log r) & \text { if } \lambda_{1}=1 \text { and } \partial \Delta / \partial \lambda=0 \text { at } \lambda_{1}=1 \\
O(1) & \text { if no zeros of } \Delta \text { in the strip } \\
& \text { and } \partial \Delta / \partial \lambda \neq 0 \text { at } \lambda_{1}=1\end{cases}
\end{aligned}
$$

The conditions for bounded/singular behaviour are more complicated than before and are found in the following manner. From equation (48) this condition is defined by the locus of points along which the logarithmic singularities occur (as long as no other root occurs in the strip). Therefore, $\partial \Delta / \partial \lambda$ is found and $\lambda_{1}=1$ is substituted into the resulting expression which, when evaluated to zero, gives the curve delineating the bounded/singular regions. As an illustration consider the special case of $\alpha_{1}=\pi$ and $\alpha_{2}=\pi / 2$ (Fig. 5a). Following the above procedure, $-2(1+\alpha)^{2}=0$ is obtained as the locus and note that the point $\alpha=-1$ satisfies this condition. Results for this example case are given in Fig. 5b. As there are now four independent physical variables in the problem $\left(\alpha_{1}, \alpha_{2}, \alpha\right.$ and $\left.\beta\right)$ a comprehensive display of the characteristic solution to the problem is not feasible. 


\section{INTERFACE CRACK}

A special case of the above problem is when two bodies are bonded along a common, straight interface, save over a small region where the bond fails, leaving an interface crack (Fig. 4f). This may be studied using the Bogy procedure by specializing each of the wedges to a half-plane, which gives rise to some interesting and surprising results. In particular, it is found that, with external loading, which would normally cause opening of the crack tips, in mode I, they actually remain closed. If an attempt is made to model the crack as if it were open to the tips, it is found that there is local implied interpenetration, as found by England [21]. In order to get round this problem a complete solution to the problem of a finite crack in an infinite plane was first provided by Comninou [22], who assumed that the crack faces adjacent to the crack tip were pressed together, giving rise to a local contact pressure. The solution she derived implies, in fact, a singular distribution of contact pressure, so that there is a mode I compression field in $(a, b)$, whereas ahead of the crack tip the material sees mode II loading. This solution was subsequently refined by Gautesen and Dundurs [23]. It seems counterintuitive, even though it is mathematically rigorous. The contact length adjacent to the crack tip is very small indeed (although it may be quite large if there is also remote mode II loading [24]) and a little further out from the crack tip the nominal stress fields look very much like the conventional ones present at an open crack tip. If the process (plastic) zone extends to a region in which the local stress field looks like that of an open crack tip, the refined solution may not be needed. This question will be addressed in more detail later, where the application of nested asymptotes to help address this problem is described.

The classical 'bilateral' (traction-free faces) solution to the interface crack problem gives rise to stresses present along the line of the interface of the form [25]

$$
\sigma_{y y}+\mathrm{i} \tau_{x y}=\frac{K}{\sqrt{r}}\left(\frac{r}{a}\right)^{\mathrm{i} \varepsilon}
$$

where $r$ is the distance from the crack tip, $a$ the crack half-width, $K$ a complex quantity analogous to the stress intensity factor and $\varepsilon$ is a dimensionless bimaterial parameter defined by

$$
2 \pi \varepsilon=\log \left(\frac{1+\beta}{1-\beta}\right)
$$

and $\beta$ is Dundurs' parameter, defined above. The corresponding discontinuity in displacement across the crack faces is given by

$$
\Delta v+\mathrm{i} \Delta u=\frac{\left[\left(\kappa_{1}+1\right) / \mu_{1}+\left(\kappa_{2}+1\right) / \mu_{2}\right] K(r / a)^{\mathrm{i} \varepsilon} \sqrt{r}}{2(1+2 \mathrm{i} \varepsilon) \cosh (\pi \varepsilon)}
$$

This solution implies an oscillatory displacement field with points of interpenetration, which cannot be physically correct. The exact solution implies that, over a short distance attached to the crack ends, the crack faces are pressed together, so that there is no mode I singularity. There is, however, a mode II singularity ahead of the crack tip.

The most important feature of this solution is the nature of the 'physically incorrect' bilateral solution (the one ignoring the possibility of crack face contact). Although this predicts contact at a number of points within the crack, the interpenetration zones are widely separated. If a new angle, $\theta_{i}$, is defined by the relation

$$
\theta_{i}=\arg \left(\Delta u_{y}+\mathrm{i} \Delta u_{x}\right)
$$

then equation (51) gives

$$
\theta_{i}=\arg (K)+\varepsilon \log \left(\frac{r}{a}\right)-\tan ^{-1}(2 \varepsilon)
$$

Interpenetration zones are defined by $\cos \left(\theta_{i}\right)<0$, i.e.

$$
\left(2 n-\frac{3}{2}\right) \pi<\theta_{i}<\left(2 n-\frac{1}{2}\right) \pi
$$

where $n$ is any integer. This shows that the relative distance between any adjacent pair of contact regions is in the ratio $[\exp (2 \varepsilon / \pi)]^{4}$. This is a very large number indeed: its minimum possible value is about $3.9 \times 10^{15}$, so that this implies that, if there is a point of interpenetration of order of the crack length, the next one down in size is truly minute, so minute as to be swamped by a plastic region at the crack tip for any practically important load. This illustrates that, while the classical bilaterally defined solution is formally incorrect, in practice it is of good quality, with physical inconsistencies occurring only within the region where, in any case, the material is not in an elastic state and therefore the solution is invalid. Details of the argument, and its extension to configurations loaded remotely in shear, are given in reference [25].

\section{FRICTIONAL CONTACTS}

The problem of interfaces between components in relative motion is now discussed. The intended application of this class of solution is to components in contact but suffering relative tangential motion in the presence of friction. In particular, the results to be derived may be applied to components suffering fretting damage, providing that the relative motion is not sufficiently damaging for wear to change the local profile, which remains unchanged. If the coefficient of friction is sufficiently high for the bodies to adhere, the problem becomes that of a monolithic contact, with a solution given either by the Williams solution if the bodies have the same elastic constants or the Bogy solution if they are dissimilar. If the coefficient of friction is not 

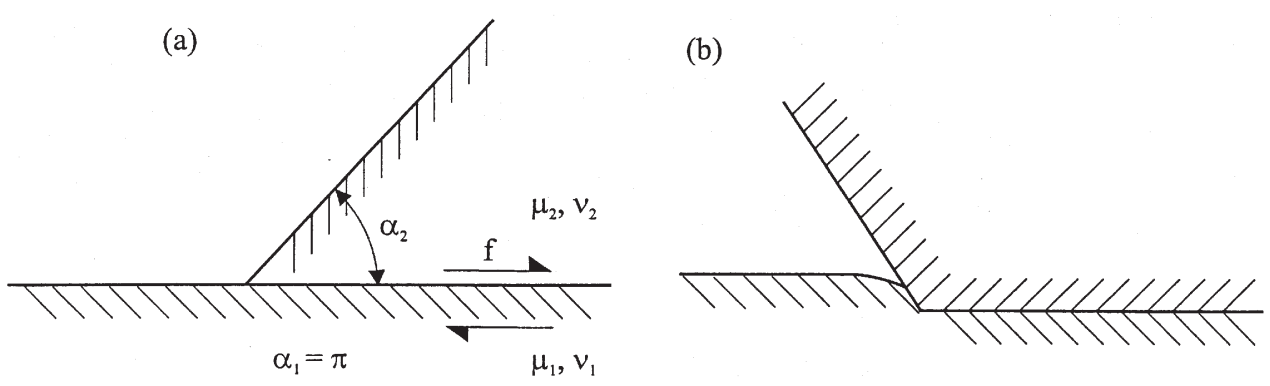

Fig. 6 Asymptotic frictional contact problem for sliding semi-infinite wedges

sufficiently high to prevent local slip, the situation at the contact edge may be represented by two wedges with mixed interfacial boundary conditions; there must be continuity in the $\theta$ direction displacement and the shearing traction must be equal in magnitude to the product of the coefficient of friction, $f$, and the direct traction at that point (Fig. 6a):

$$
\begin{aligned}
& u_{\theta 1}=u_{\theta 2} \\
& \left|\tau_{r \theta}\right|=-f \sigma_{\theta \theta}, \quad \sigma_{\theta \theta}<0
\end{aligned}
$$

This problem was tackled by Gdoutos and Theocaris [26] and also by Comninou [27], which should be consulted for full details. In order to reduce the number of independent variables, body 1 is assumed to be a half-plane $\left(\alpha_{1}=-\pi\right)$. A further point to bear in mind is that the nature of the solution depends on the direction in which slippage occurs, so that the following sign convention is used:

$$
\begin{gathered}
f>0 \Rightarrow \text { wedge }\left(\alpha_{2}\right) \text { slipping in the }+ \text { ve } x \text { direction } \\
\text { (away from the corner) } \\
f<0 \Rightarrow \text { wedge }\left(\alpha_{2}\right) \text { slipping in the }- \text { ve } x \text { direction } \\
\text { (towards the corner) }
\end{gathered}
$$

Each of the papers cited deals also with the case where the bodies may be elastically dissimilar, when, in the same nomenclature used above (Fig. 5a), the eigenequation corresponds to the determinant of the boundary value problem given by

$$
D\left(f, \alpha_{2}, \alpha, \beta ; \lambda\right)=8(1+\lambda) \sin (\lambda \pi) F\left(f, \alpha_{2}, \alpha, \beta ; \lambda\right)
$$

where

$$
\begin{aligned}
& F\left(f, \alpha_{2}, \alpha, \beta ; \lambda\right) \\
& =(1+\alpha) \cos \lambda \pi\left(\sin ^{2} \lambda \alpha_{2}-\lambda^{2} \sin ^{2} \alpha_{2}\right) \\
& +\frac{1}{2}(1-\alpha) \sin \lambda \pi\left(\sin 2 \lambda \alpha_{2}+\lambda \sin 2 \alpha_{2}\right) \\
& +f \sin \lambda \pi\left[(1-\alpha) \lambda(1+\lambda) \sin ^{2} \alpha_{2}\right. \\
& \left.\quad-2 \beta\left(\sin ^{2} \lambda \alpha_{2}-\lambda^{2} \sin ^{2} \alpha_{2}\right)\right]
\end{aligned}
$$

The case is also recorded where the two components have the same elastic constants, which occurs more frequently in practice, so that equation [54] becomes

$$
\begin{aligned}
F\left(f, \alpha_{2}, 0,0 ; \lambda\right)= & \cos \lambda \pi\left(\sin ^{2} \lambda \alpha_{2}-\lambda^{2} \sin ^{2} \alpha_{2}\right) \\
& +\frac{1}{2} \sin \lambda \pi\left(\sin 2 \lambda \alpha_{2}+\lambda \sin 2 \alpha_{2}\right) \\
& +f \sin \lambda \pi \lambda(1+\lambda) \sin ^{2} \alpha_{2}
\end{aligned}
$$

It is noteworthy that, in the case where one of the wedges is an elastic half-plane and the other wedge represents the edge of a finite contacting body, the gradient of the displacement field immediately exterior to the contact edge is singular, i.e. it has a gradient normal to the free surface. It follows that the solution described above is strictly valid only when the punch has an internal angle which is $\pi / 2$ or less, if additional contact exterior to the contact face along the inclined flank (Fig. 6b) is to be avoided. A related problem has been studied by Adams [28] who solves explicitly for the contacting length. This is normally quite small and, providing the plastic zone is larger in size than the contact length, the validity of a single asymptotic solution in which its presence is neglected will not be invalidated. Figure 7 shows a plot of the order of singularity, $\lambda-1$, against the wedge angle, $\alpha_{2}$, when $\alpha=\beta=0$ for sample coefficients of friction. These contours are found by plotting $F\left(f, \alpha_{2}, 0,0 ; \lambda\right)=0$ [from equation (58) above] for various $\alpha_{2}$ and constant $f$. Details of the interpretation of this figure can be found in Mugadu et al. [29]. It is clear that, for a given contact angle, $\alpha_{2}$, the singularity at the corner is stronger when the frictional traction is directed towards the contact corner than when it is directed away. Also, it is noteworthy that, for strong coefficients of friction and contact angles around $100^{\circ}$, singularities that are slightly stronger than the square root can be anticipated.

\subsection{Mode III frictional contact}

Suppose that two elastically similar wedges are pressed together and slid in the $z$ direction to produce what may be thought of as mode III loading, i.e. where the frictional shearing traction is directed parallel with a 


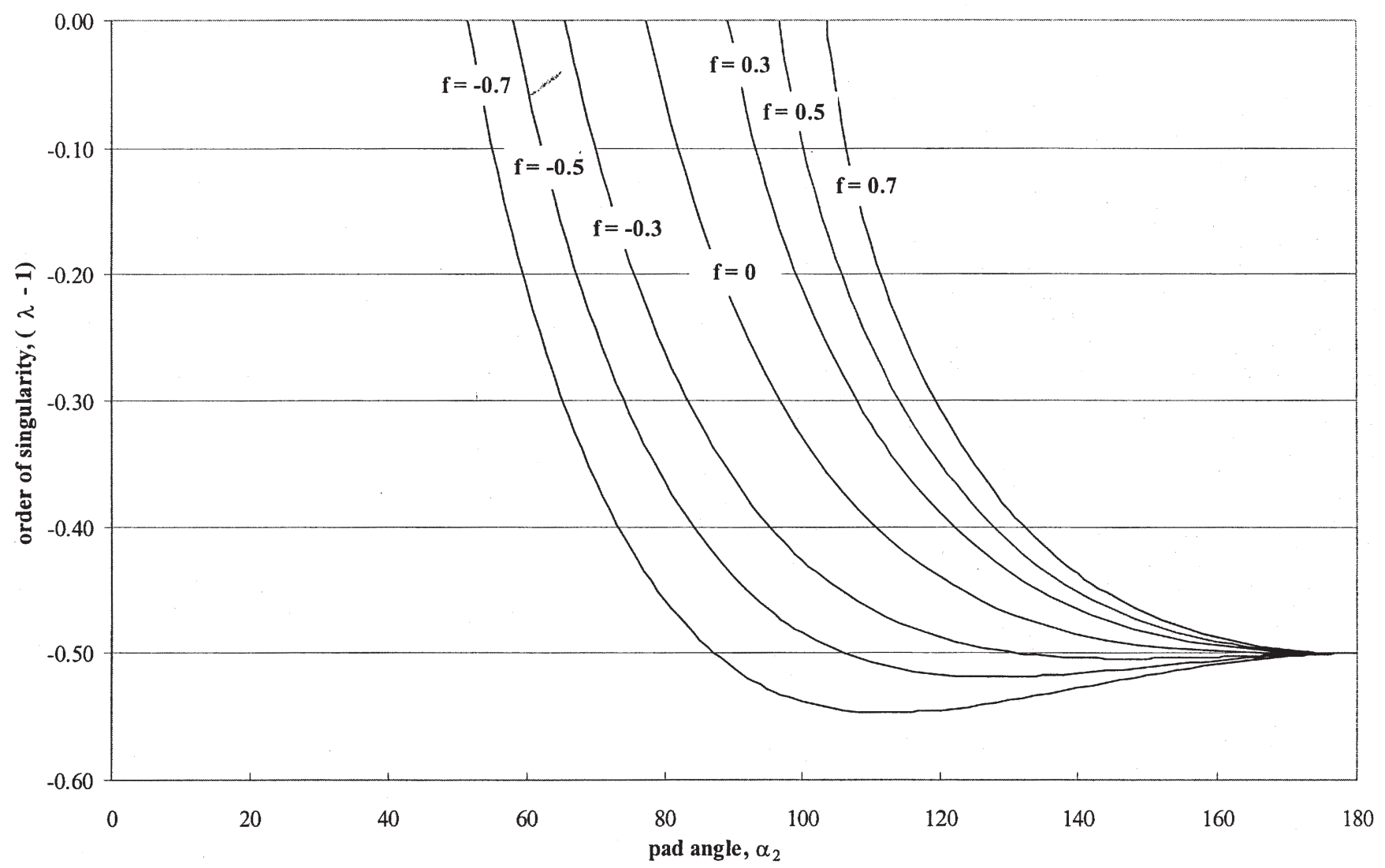

Fig. 7 Plot showing the order of singularity, $\lambda-1$, against the pad angle, $\alpha_{2}$, as a function of the coefficient of friction, $f$

tangent to the contact edge, e.g. as when a ball is pressed into a block and twisted or as shown schematically in Fig. 8a. This problem is of practical interest in determining the local behaviour of, for example, a shrink-fitted shaft subject to torsion, as it describes the stress state when the shaft emerges from the pulley or wheel into which the shrink has been made (Fig. 8b). This problem differs fundamentally from the 'mode II' frictional contact described above, in that slip in the $z$ direction produces an antiplane problem that is uncoupled from the in-plane component of loading, which here is the contact pressure, $\sigma_{\theta \theta}$. It follows that, as $\tau_{r \theta}$ is zero on the slipping faces of the wedges, the in-plane solution corresponds exactly to that of frictionless loading and that this part of the problem may be solved separately. When once this has been done, the effect of the antiplane traction may be found by considering a straightforward boundary value problem for each of the wedges, in isolation. The free boundary, $\theta=\alpha_{2}$, is devoid of tractions:

$$
\sigma_{\theta \theta}=\tau_{r \theta}=0, \quad \theta=\alpha_{2}
$$

while on the interface boundary, $\theta=0$, the direct traction, $\sigma_{\theta \theta}(r)$, is specified by the eigenfunction for the frictionless contact problem, and $\tau_{z \theta}=f \sigma_{\theta \theta}$.
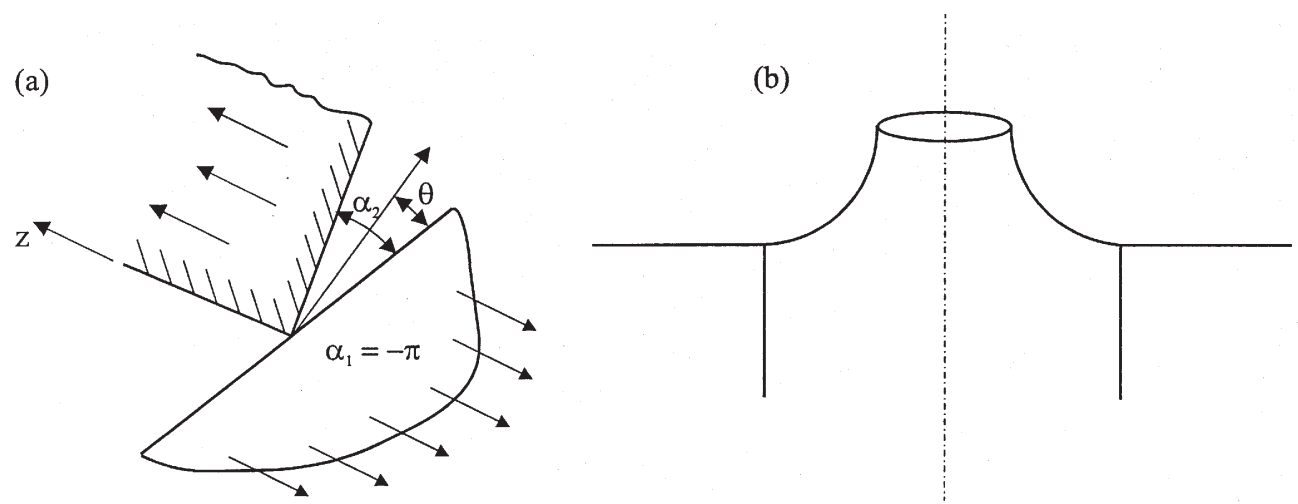

Fig. 8 Mode III frictional slip: (a) example of where it arises and (b) idealization of the asymptotic solution 
It is possible to extend this approach to the case of mixed mode II and III loadings. It will be assumed that the two semi-infinite wedges are being slid, relative to each other, along a line making an angle $\phi$ with the $r$ direction, and this solution is currently under investigation.

\section{EMBEDDED ASYMPTOTIC SOLUTIONS}

A number of situations arise in which the asymptotic solutions developed above are insufficient to describe the behaviour of the local stress state, usually because the geometry of the problem in the immediate neighbourhood of the discontinuity is not that implied by the mathematical description. The most obvious case where this may be so is at the root of a V-notch or crack, where there will normally be a finite radius present, and it will not be atomically sharp. Clearly, the presence of a finite radius at the crack root will formally invalidate the asymptotic solution, but, if the radius is very small, it seems intuitively correct that the asymptotic solution will be recovered, for practical purposes. However, how big can the crack tip radius be? A simple answer to this question is to say that, providing that the radius is small compared with the size of the process (plastic) zone, then its presence will not be felt, and this is the usual criterion employed. Thus the range of loads that can be supported by a cracked component and for linear elastic fracture mechanics to be valid may be found in the following way: the upper bound to the load is imposed by the requirement that the boundary of the process zone must be well within the region in which the singular $\left(K_{\mathrm{I}}\right)$ crack tip field dominates the state of stress, and the full field is itself geometry-dependent. On the other hand, the lower bound is dictated by the requirement that the plastic zone must be large compared with the radius of any crack tip radius present. If the crack is extending by fatigue, and hence the component is subjected to a fluctuating load, these bounds will set bounds to the upper and lower loads respectively and, if these bounds are not satisfied, predictions based on linear elastic fracture mechanics theory will be unsafe.

\subsection{Crack tip and notch root solutions (finite root radius)}

Recently, a more sophisticated solution to the question of the tolerable crack tip radius has been devised [30], which employs a semi-infinite rounded slot solution to represent the crack end and which is embedded within the singular crack tip solution. The spirit of the procedure is that a nested set of asymptotic solutions is developed. There is an outer 'full-field' solution, as previous described, and, moving in towards the singularity, an asymptotic solution (in the case cited, a conventional crack tip stress intensity solution, which is scaled by the stress intensity factor). This element of the analysis may be thought of as collocating a semi-infinite crack solution into the finite crack problem. Now, moving inwards again, the outer solution for the semi-infinite slot solution is crack-like in behaviour, so this may now be embedded within the semi-infinite crack solution, using the stress intensity factor as the scaling quantity. Explicit solutions monitoring the increasing discrepancy between the crack solution and the slot solution may be found, and hence these provide a more precise measure of the minimum plastic (process) zone radius.

The innermost solution under consideration is that for a semi-infinite slot having a radiused end, recently developed by Filippi et al. [31]. This paper should be consulted for full details, but here the results found will simply be recorded. The paper itself deals with a rounded-root, straight-sided notch, so the geometry is first specialized by making the root sides parallel. Note that there are two solutions to this problem, one that is symmetrical in nature, so that direct stresses arise on the line of symmetry but with no shearing tractions (mode I in fracture mechanics jargon), while the other is antisymmetrical. Here only the shear stress is non-zero on the line of symmetry and this is therefore 'mode II'. The solutions are, of course, elastic, and hence may be superposed to obtain any mode mixity required.

A generalization of the procedure described above is possible for the case when the basic singular solution refers to a sharp notch. Exactly the same philosophy of 'nesting' the solutions applies and the procedure has again been made possible by the results found in Filippi et al. [31].

\subsection{Complete contact solution (finite end radius)}

The solution for the frictional slipping of wedges described above assumes that the contact corner is extremely sharp and, of course, in practice this is unlikely to be achieved. In the spirit of the solution described above, the state of stress in the neighbourhood of the edge of a semi-infinite punch having a rounded profile has recently been deduced. Interest is principally on a punch where the faces of the punch are perpendicular to the end face, and therefore an 'outer asymptote' is needed in the form of a semi-infinite square-ended punch. This could be found in one of two ways: either a start could be made from the general solution for two wedges, specializing one to a half-plane and the other to a quarter-plane, or, if the punch is rigid, the standard solution could be employed for a finite rigid, squareended punch and an asymptotic expansion performed at the punch corner [32].

\subsection{Interface crack}

The problem of a crack present at an interface between elastically dissimilar materials introduces issues that are 
counterintuitive; it does not seem reasonable that the application of a remote tensile force leaves the crack tips closed (and hence giving rise to no mode I loading), but inducing a mode II singularity. In fact, there is a mode I singularity, but it occurs in the form of a local contact pressure between the crack faces. The question that arises is: "What are the implications of these observations for the behaviour of the local stress field and, in turn, what are its influences on the behaviour of the local process zone, and hence crack extension?' Further notes on this problem are included in reference [25].

\section{CONCLUSION}

A comprehensive review has been given of the origins of asymptotic procedures in stress analysis. It has been shown that the elastic stress state adjacent to a notch tip can be accurately defined by a dominant singular term solution whose characteristics depend on the nature of the external loading (mode I, II and III loadings). The corresponding generalized stress intensity factor $\left(K_{\mathrm{I}}, K_{\mathrm{II}}\right.$ or $\left.K_{\mathrm{III}}\right)$ is then used to scale the asymptotic solution to the local stress state. Additionally, the solutions may be combined using the principle of superposition to give a 'mixed mode' notch tip elastic stress field. Lastly, the presence of a stress intensity factor leads to local yielding so that a process or plastic zone arises. The limitations of the asymptotic procedure in quantifying the stress state, i.e. small scale yielding, have been defined.

Bonded elastically dissimilar components were then considered. Once again it was possible to deduce the nature of the singular elastic stress state adjacent to the apex. However, unlike the crack and notch configurations mentioned above, there is one singular solution corresponding to a given set of parameters. Furthermore, the singular solution is neither symmetric nor antisymmetric. Additionally, for this class of problem, the eigenvalues associated with the boundary value problem may be complex, giving rise to oscillating singularities. A frictional interface was then introduced; the analysis is analogous to that of the bonded wedges. It is noteworthy that, for this class of problem, the order of singularity also depends on the relative direction of slip. Additionally, out-of-plane slip (mode III in fracture mechanics jargon) was considered, allowing 'mixed mode' frictional behaviour to be probed.

Lastly, it is appreciated that the asymptotic analyses of elastic stress fields assume the edges to be atomically sharp. However, in practice, there will be a local radius. The question then is: 'How large can the radius be for the asymptotic solution to still hold?' This is answered using the concept of nested asymptotes, the outer asymptote being the semi-infinite asymptotic solutions described above and the inner asymptote being that appropriate to a semi-infinite rounded geometry. This procedure has been applied to the crack tip, the Vnotch and the slipping asymptotic solutions.

\section{REFERENCES}

1 Broek, D. Elementary Engineering Fracture Mechanics, 1984 (Martinus Nijhoff, The Hague, The Netherlands).

2 Broek, D. The Practical Use of Fracture Mechanics, 1988 (Kluwer, Dordrecht, The Netherlands).

3 Ewalds, H. L. and Wanhill, R. J. H. Fracture Mechanics, 1986 (Delftse Uitgevers Maatschappij, Delft, The Netherlands).

4 Gdoutos, E. E. Fracture Mechanics, 1993 (Kluwer, Dordrecht, The Netherlands).

5 Gdoutos, E. E. Fracture Mechanics Criteria and Applications, 1990 (Kluwer, Dordrecht, The Netherlands).

6 Kanninen, M. F. and Popelar, C. H. Advanced Fracture Mechanics, 1985 (Oxford University Press, Oxford).

7 Broberg, K. M. Cracks and Fracture, 1999 (Academic Press, Cambridge).

8 Aliabadi, M. H. and Rooke, D. P. Numerical Fracture Mechanics, 1991 (Kluwer, Dordrecht, The Netherlands).

9 Westergaard, H. M. Bearing pressures and cracks. Trans. ASME, J. Appl. Mechanics, 1937, 6, A49-A53.

10 Griffith, A. A. The phenomena of rupture and flow in solids. Phil. Trans. R. Soc. Lond., 1921, A221, 163-198.

$11 \mathrm{Sih}$, G. C. Strain energy density factor applied to mixed mode crack problems. Int. J. Fracture, 1974, 10, 305-321.

12 Sih, G. C. Mechanics of Fracture Initiation and Propagation, 1999 (Kluwer, Dordrecht, The Netherlands).

13 Plank, R. and Kuhn, G. Fatigue crack propagation under non-proportional mixed mode loading. Engng Fract. Mechanics, 2001, 68, 53-76.

14 Williams, M. L. Stress singularities resulting from various boundary conditions in angular plates in extension. $J$. Appl. Mechanics, 1952, 19, 526-528.

15 Barber, J. R. Elasticity, 1992 (Kluwer, Dordrecht, The Netherlands).

16 Tur, M., Fuenmayor, J., Mugadu, A. and Hills, D. A. On the analysis of singular stress fields: Part 1: finite element formulation and application to notches. J. Strain Analysis, 2002, 37(5), 437-444.

17 Leguillon, D. Strength or toughness? A criterion for crack onset at a notch. Eur. J. Mechanics, A/Solids, 2002, 21, 61-72.

18 Bogy, D. B. Edge-bonded dissimilar orthogonal elastic wedges under normal and shear loading. J. Appl. Mechanics, 1968, 35, 460-466.

19 Dundurs, J. Discussion of reference [18]. J. Appl. Mechanics, 1969, 36, 650-652.

20 Bogy, D. B. Two edge-bonded elastic wedges of different materials and wedge angles under surface tractions. $J$. Appl. Mechanics, 1971, 38, 377-386.

21 England, A. H. A crack between dissimilar media. J. Appl. Mechanics, 1965, 32, 400-402.

22 Comninou, M. The interface crack. J. Appl. Mechanics, 1977, 44, 631-636.

23 Gautesen, A. K. and Dundurs, J. The interface crack in a tension field. J. Appl. Mechanics, 1987, 54, 93-98.

24 Comninou, M. The interface crack in a shear field. Trans. ASME, J. Appl. Mechanics, 1978, 45, 287-290. 
25 Hills, D. A. and Barber, J. R. Interface cracks. Int. J. Mech. Sci., 1993, 35(1), 27-37.

26 Gdoutos, E. E. and Theocaris, P. S. Stress concentrations at the apex of a plane indenter acting on an elastic half-plane. J. Appl. Mechanics, 1975, 42, 688-692.

27 Comninou, M. Stress singularity at a sharp edge in contact problems with friction. J. Appl. Math. Phys. (ZAMP), 1976, 27, 493-499.

28 Adams, G. G. A rigid punch bonded to a half plane. Trans. ASME, J. Appl. Mechanics, 1979, 46, 844-848.

29 Mugadu, A., Hills, D. A. and Limmer, L. An asymptotic approach to crack initiation in fretting fatigue of complete contacts. J. Mech. Phys. Solids, 2002, 50, 531-547.
30 Dini, D. and Hills, D. A. The effect of a crack-tip radius on the validity of the singular solution. Proc. Instn Mech. Engrs, Part C: J. Mechanical Engineering Science, 2004, 218, 693-701.

31 Filippi, S., Lazzarin, P. and Tovo, R. Developments of some explicit formulas useful to describe elastic stress fields ahead of notches in plates. Int. J. Solids Structs, 2002, 39, 4543-4565.

32 Sackfield, A., Mugadu, A., Barber, J. R. and Hills, D. A. The application of asymptotic solutions to characterising the process zone in almost complete frictionless contacts. J. Mech. Phys. Solids, 2003, 51, 1333-1346. 